\title{
Seeing what we know and understand: How knowledge shapes perception
}

\author{
Rasha Abdel Rahman and Werner Sommer \\ Humboldt University, Berlin, Germany
}

\begin{abstract}
Expertise in object recognition, as in bird watching or X-ray specialization, is based on extensive perceptual experience and in-depth semantic knowledge. Although it has been shown that rich perceptual experience shapes elementary perception and higher level discrimination and identification, little is known about the influence of in-depth semantic knowledge on object perception and identification. By means of recording event-related brain potentials (ERPs), we show that the amount of knowledge acquired about initially unfamiliar objects modulates visual ERP components already $120 \mathrm{msec}$ after object presentation, and causes gradual variations of activity in similar brain systems within a later timeframe commonly associated with meaning access. When perceptual analysis is made more difficult by blurring object pictures, knowledge has an even stronger effect on perceptual analysis and facilitates recognition. These findings demonstrate that in-depth knowledge not only affects involuntary semantic memory access, but also shapes perception by penetrating early visual processes traditionally held to be immune to such influences.
\end{abstract}

Accessing knowledge is essential for us to recognize and identify the large variety of objects, some more or less well known, some novel, with which we are daily confronted. However, the facets of object-related knowledge held in memory can vary considerably (e.g., Pexman, Hargreaves, Edwards, Henry, \& Goodyear, 2007). Thus, knowledge about cars can range from basic driving requirements to understanding intricate details of function. How this vast range of knowledge affects object recognition is largely unknown.

Expertise in object recognition may alter behavior (Gauthier, James, Curby, \& Tarr, 2003; Gauthier, Williams, Tarr, \& Tanaka, 1998; Tanaka, Curran, \& Sheinberg, 2005), enhance metabolism in the fusiform gyrus (Gauthier, Skudlarski, Gore, \& Anderson, 2000; Gauthier, Tarr, Anderson, Skudlarski, \& Gore, 1999), and increase the N170 component in event-related brain potentials (ERPs) (Tanaka \& Curran, 2001). These kinds of expertise typically involve not only rich perceptual experience but also profound semantic knowledge, factors that are hard to disentangle. Thus, when computergenerated, visually similar novel objects or shapes are associated with arbitrary semantic information, it affects visual discrimination (Gauthier et al., 1999) and activates a neural network related to semantic processing (James $\&$ Gauthier, 2003, 2004). Furthermore, hands-on experience with novel tools affects neural activity in extrastriate visual areas (Weisberg, van Turennout, \& Martin, 2007), as does learning novel objects in the context of different actions (Kiefer, Sim, Liebich, Hauk, \& Tanaka, 2007).
Although the above mentioned studies suggest that knowledge affects behavioral and neural aspects of object processing, they mostly used behavioral or BOLD responses, providing only limited insight into the temporal unfolding of knowledge effects. Also, it is unclear whether findings from homogeneous artificial objects, associated with arbitrary and unconnected information, would generalize to more common heterogeneous objects and connected semantic information. Therefore, the present study used a wide variety of different objects, for some of which in-depth knowledge about functional attributes was provided. Because basic semantic information may be accessed very rapidly (Thorpe, Fize, \& Marlot, 1996) - in fact, as quickly as detecting the object (Grill-Spector \& Kanwisher, 2005) — knowledge might not only affect high-level identification but may already influence early visual processing. Therefore, we exploited the high temporal resolution of ERPs to investigate the functional loci of in-depth knowledge on perception and identification.

To isolate semantic sources of expertise while controlling perceptual factors and preexisting knowledge, we manipulated the depth of knowledge about initially unfamiliar objects in a multistep learning procedure (see Figure 1). To control for changes in object perception, one participant group was tested with minimal opportunity for visual inspection of the objects during knowledge acquisition. Knowledge effects were assessed in separate test sessions with various tasks, ranging from familiarity classifications to naming. 
A

Learning Phase, Part 1

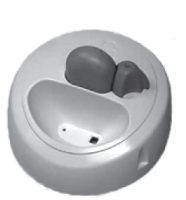

Ganosis

Real

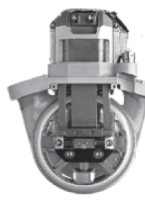

Sonocor

Fictitious

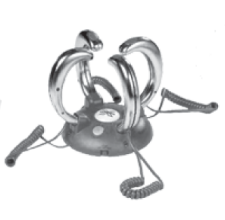

Adder Real

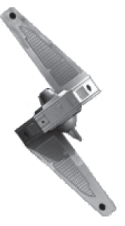

Planeo Fictitious

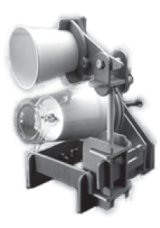

Notande Real

B

\section{Learning Phase, Part 2}

In-Depth-Knowledge Condition

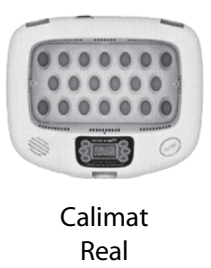

“This is an artificial incubation device for hen's eggs. Instead of letting the hens breed farmers put the eggs into this box. By adjusting temperature and humidity the breeding hen is simulated by the box very efficiently. The breeding process is even slightly faster, which saves money."

Minimal-Knowledge Condition

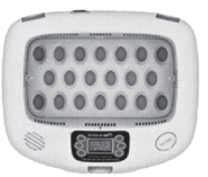

Calimat

Real

"For Italian tomato sauce, saute onions in oil in a saucepan over medium-high heat until golden brown. Add crushed tomatoes, water, tomato paste, basil, garlic, salt and pepper. Let the sauce come to a boil, and stir occasionally until desired thickness. Sauce is ready when oil rises to the top."

C

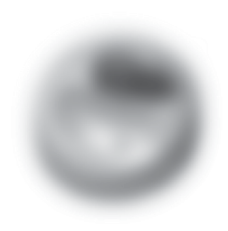

Test Phase, Blurred Example Stimuli

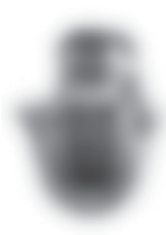

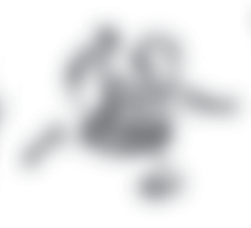

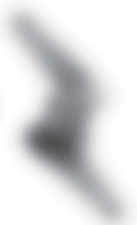

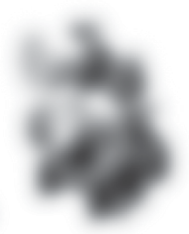

Figure 1. Example stimuli as presented in Experiments 1 and 2. The stimulus set consisted of $\mathbf{4 0}$ rare objects, such as historic tools and gadgets, with largely unknown functions. (A) Examples for object pictures and task-relevant information to be memorized in the first part of the learning sessions. (B) English examples for a story with in-depth expert knowledge (in-depth-knowledge condition) and an unrelated story (minimal-knowledge condition), as presented in the second part of the learning session. While the stories were played, the objects were presented on the screen (Group 1 of Experiment 1), or only the associated information (name and real vs. fictitious) was presented in the absence of the object picture (Group 2 of Experiment 1 and Experiment 2). (C) Examples of blurred versions of the images, as presented in Experiment 2.

We focused on ERP components associated with meaning access and low-level visual perception. The N400 component is most pronounced around $400 \mathrm{msec}$ after stimulus onset. Its amplitude increases with the depth of semantic processing associated with meaningful stimuli such as words and objects (Kutas \& Hillyard, 1980; Kutas, Van Petten, \& Kluender, 2006). Therefore, we expected an amplitude modulation of the N400, due to in-depth knowledge.
The P1 component peaks about 100 to $130 \mathrm{msec}$ after stimulus onset and reflects processing of visual object features in extrastriate cortex (Di Russo, Martínez, Sereno, Pitzalis, \& Hillyard, 2001). P1 amplitude is sensitive to spatial attention (Hillyard \& Anllo-Vento, 1998) and other cognitive processes such as facial expression analysis (Meeren, van Heijnsbergen, \& de Gelder, 2005). Assuming that increased knowledge about the meaning of object features facilitates perceptual analysis or visual 
integration of these features, we expected a decrease in the amount of the corresponding neural activity, reflected in P1 magnitude.

\section{EXPERIMENT 1}

\section{Method}

Participants. Participants were 40 native German speakers (32 women; mean age, 24.1 years; age range, 19-41), who had given informed consent.

Materials. Stimuli were pictures of 40 rare objects with largely unknown functions (neither pictures nor names revealed any meaningful information about functional properties), and 20 well-known objects. Forty spoken stories containing functional information about objects and 20 cooking recipes were recorded (mean durations were 18.3 vs. $18.6 \mathrm{sec}$ )

Procedure. The learning session consisted of two parts. In Part 1, lasting about $45 \mathrm{~min}$, participants were familiarized with all rare objects and learned the task-relevant information (names and whether the object was real or fictitious), terminating with a test of the taskrelevant knowledge.

In Part 2, lasting on average $1.5 \mathrm{~h}$, short stories were presented while either the object images (Group 1, $n=20$ ) or only the written task-relevant information associated with the object was shown on the screen (Group 2, $n=20$ ). For half of the objects, the stories contained information about the object's function (in-depth-knowledge condition; see Figure 1). For the other objects, unrelated cooking recipes were presented (minimal-knowledge condition), controlling for unspecific effects of attention, fatigue, and so on. Presentation times of objects in the two conditions were identical. The assignment to the in-depth-knowledge and minimal-knowledge conditions was counterbalanced across participants within a group; therefore all objects were assigned equiprobably to each condition. All stories were presented three times. Object-related stories were always assigned to the same objects; recipes were randomly assigned to different objects at each presentation, precluding the formation of fixed associations. This part terminated with the same knowledge test as did Part 1

Following Part 1, naming latencies $(M=1,244 \mathrm{msec})$ were slower than semantic decisions $(M=972 \mathrm{msec})[F(1,39)=63.3$ $p<.001]$. After knowledge acquisition this difference increased because naming was slowed to $M=1,382 \mathrm{msec}[F(1,38)=34.1$, $p<.001]$, whereas there was no significant change for the classification task $(M=947 \mathrm{msec})(F=1.7)$. Slowing of naming was more pronounced for the in-depth-knowledge than for the minimalknowledge condition $(M=1,407$ vs. $1,357 \mathrm{msec})[F(1,39)=4.9$, $p<.05]$. These task- and knowledge-dependent changes between Parts 1 and 2 gave rise to a three-way interaction $[F(1,38)=3.9, p=$ $.053]$, indicating that the acquisition of in-depth knowledge affects the retrieval of other pieces of knowledge.

Test sessions took place two to three days after learning. First, the objects (Group 1) or names (Group 2) were presented in print and participants gave all the information they remembered. Then the newly learned objects were presented, alternating randomly with well-known real and fictitious objects (e.g., sofa vs. gingerbread house). Three tasks were employed, none of which required the retrieval of in-depth knowledge, nor was this knowledge mentioned in the instructions. In the familiarity task, participants classified objects as "well-known" or "newly learned" by buttonpresses. In the semantic task, participants verbally indicated whether objects were "real" or "fictitious," and in the speech production task, objects were named. Objects were presented three times in each task, resulting in 60 trials per condition and task. Tasks alternated blockwise in counterbalanced order.

A trial began with a fixation cross at the center of a light gray screen for $0.5 \mathrm{sec}$, followed by a picture, disappearing with the response, or after $3 \mathrm{sec}$. The next trial began $1 \mathrm{sec}$ later.

Data recording and analysis. In the test sessions, the electroencephalogram (EEG) was recorded with $\mathrm{Ag} / \mathrm{AgCl}$ electrodes from 56 sites according to the extended 10-20 system, referenced to the left mastoid, and at a sampling rate of $500 \mathrm{~Hz}$ (bandpass $0.032-70 \mathrm{~Hz}$ ). Electrooculograms were recorded from the left and right outer canthi and beneath and above the left eye. Electrode impedance was kept below $5 \mathrm{k} \Omega$. Prototypical eye movements for later artifact correction were obtained in a calibration procedure. Offline, the continuous EEG was transformed to average reference and low-pass filtered at $30 \mathrm{~Hz}$. Eye movement artifacts were removed with a spatiotemporal dipole modeling procedure using the BESA software (Berg \& Scherg, 1991). Remaining artifacts were eliminated with a semiautomatic rejection procedure. Error- and artifact-free data were segmented into epochs of $2.5 \mathrm{sec}$, starting $100 \mathrm{msec}$ prior to picture onset, with a $100-\mathrm{msec}$ prestimulus baseline interval. Global field power (GFP; Lehmann \& Skrandies. 1980) was computed as overall activity at each time point across the 56 scalp electrodes.

Amplitude differences were assessed with repeated measures ANOVAs. Huyhn-Feldt corrections were applied when appropriate. For the analyses of topographical distributions, the difference waveforms for each participant were scaled to the individual GFP of each participant.

\section{Results and Discussion}

Because results were very similar in both participant groups, we collapsed their data (see Figures 2 and 3 ). Response times (RTs) and error rates (see Figure 2) increased from familiarity over semantic classification to naming $[F(2,76)=171.1$ and 17.3 , respectively, $p \mathrm{~s}<$ $.001]$. However, there was no main effect of in-depth versus minimal knowledge and no interaction $\left(F_{\mathrm{S}}<2.3\right)$. Thus, knowledge effects in performance that had been present immediately after acquisition vanished after several days of consolidation.

In ERPs, the most prominent knowledge effect was an increased posterior negativity in the latency range of the N400 (300-500 msec). We conducted a three-way ANOVA on mean amplitudes for this time window with factors electrode (56 electrodes), ${ }^{1}$ task (familiarity classification, semantic classification, naming) and knowledge condition (in-depth knowledge, minimal knowledge, well-known objects), and the between-subjects factor group. This analysis revealed main effects of task $[F(110,4180)=5.71, p<.0001]$ and knowledge condition $[F(110,4180)=25.61, p<.0001]$, and an interaction of task, knowledge condition, and group $[F(220,8360)=$ 2.03, $p<.006]$, mainly due to stronger knowledge effects in Group 2. Separate comparisons revealed a difference between the in-depth-knowledge and minimal-knowledge conditions $[F(55,2090)=14.49, p<.001 ; F(1,38)=$ $30.0, p<.001$, when tested only at electrode sites $\mathrm{Pz}$, P7, and P8] (see Figure 3) and between common objects and both the in-depth-knowledge $[F(55,2090)=13.89$, $p<.001 ; F(1,38)=36.1, p<.001$, at sites $\mathrm{Pz}, \mathrm{P} 7$, and $\mathrm{P} 8]$ and the minimal-knowledge $[F(55,2090)=45.2, p<$ $.001 ; F(1,38)=138.8, p<.001$, at sites Pz, P7, and P8] conditions.

For the P1 time window (100-150 msec), ANOVAs on mean amplitudes across all electrodes yielded main effects of task $[F(110,4180)=2.32, p<.018]$ and knowledge condition $[F(110,4180)=6.67, p<.0001]$, reflecting an overall higher amplitude in the minimal-knowledge condition. Separate comparisons revealed a difference between 

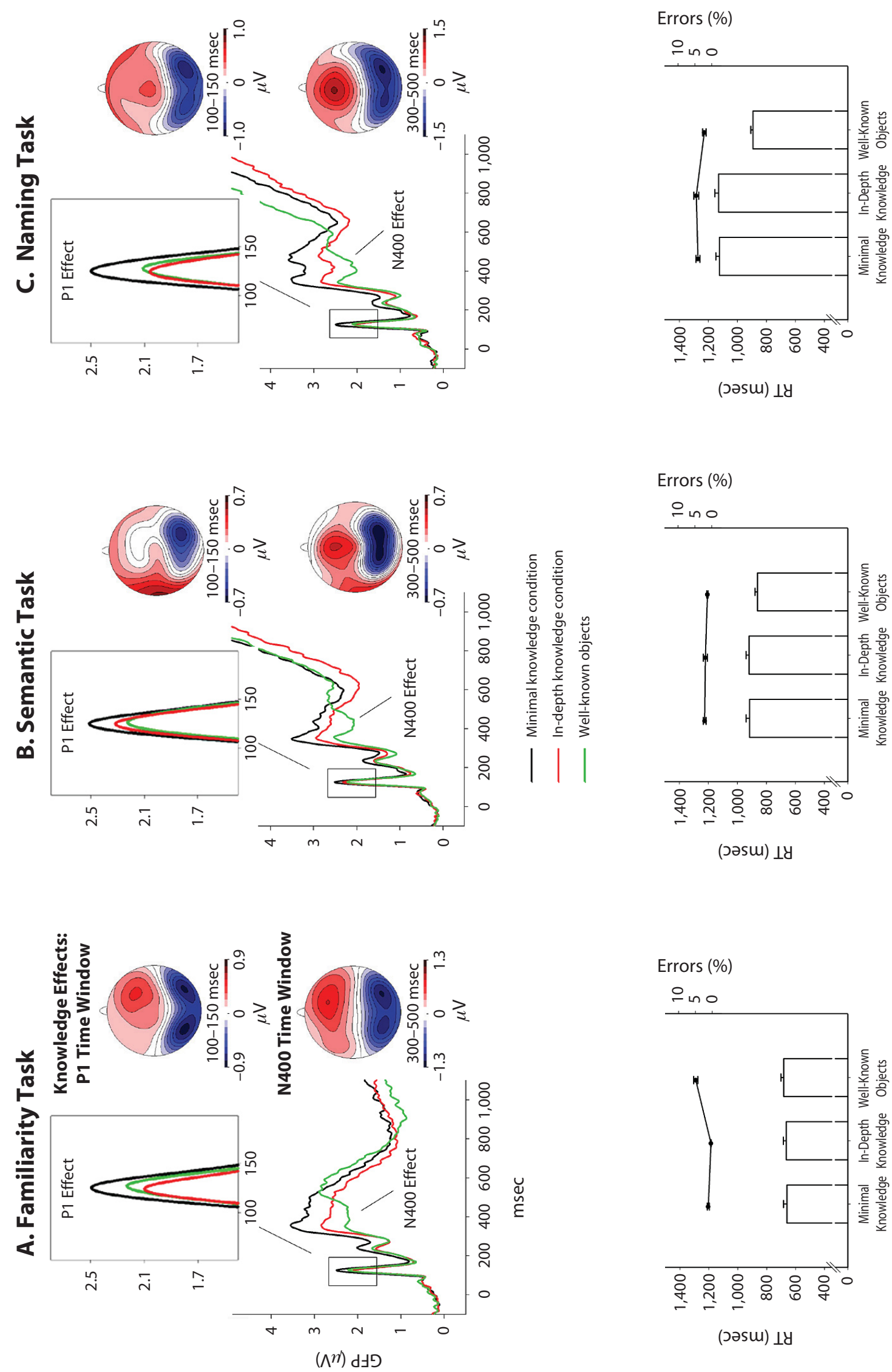

(Jәsw) $\perp$ y

Errors (\%)

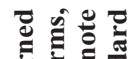

ฮ윻

势

它完

ํㅡㅇ 흥

블

远苛

赵运

的哭

记

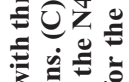
氙

颌

可

늘
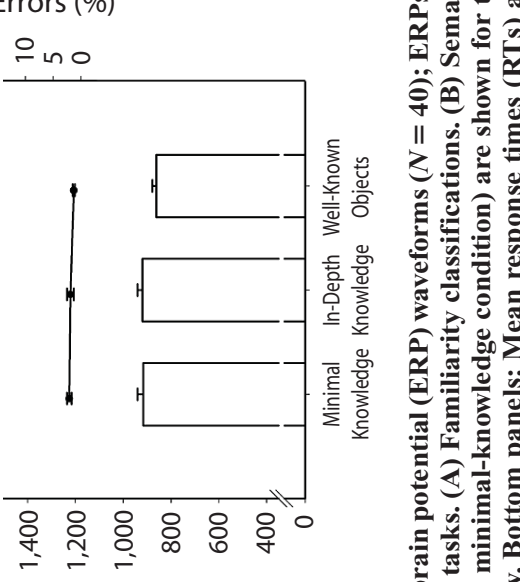

(כәsw) $\perp$ प

듕ㅎํ

宇夗

II

ย ฮิ

范

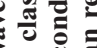

¿

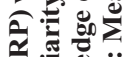

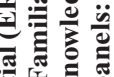

린돈

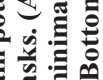
콣

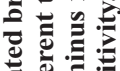

른

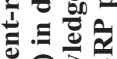

Errors (\%)

응

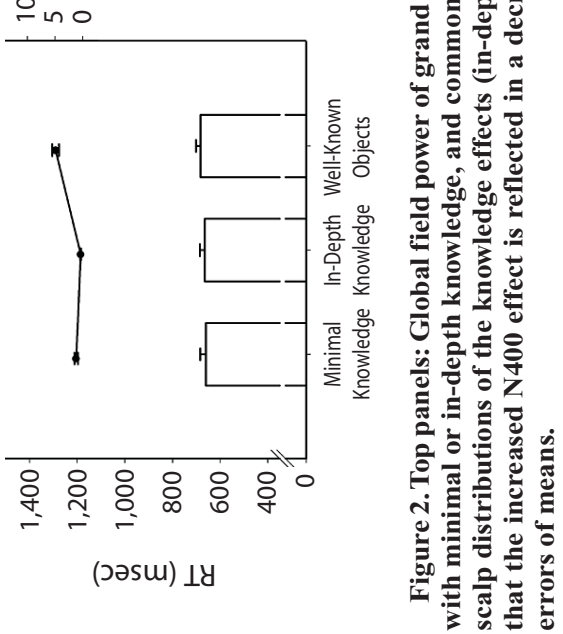



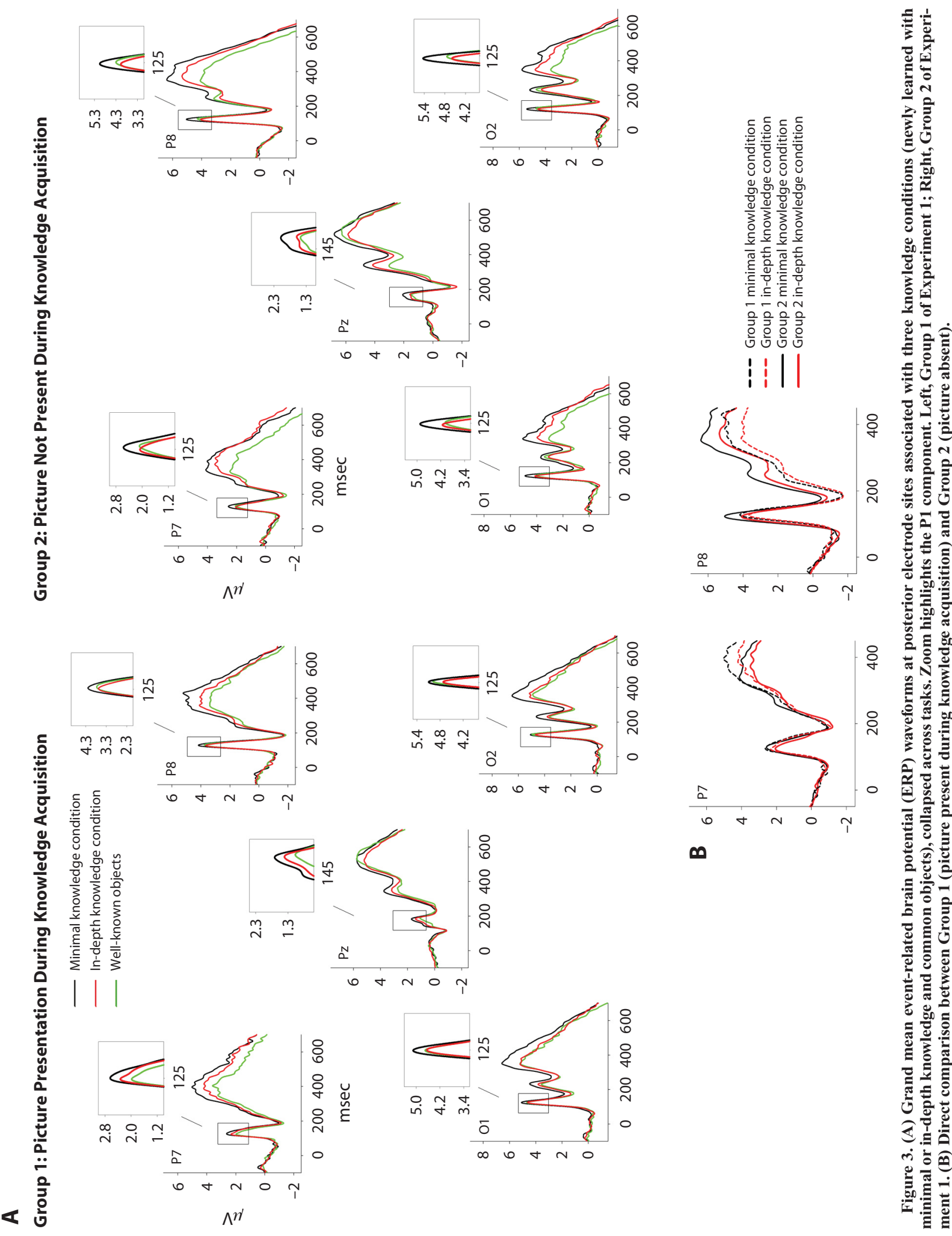

急

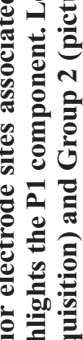

:

ส

空

윰

웡 

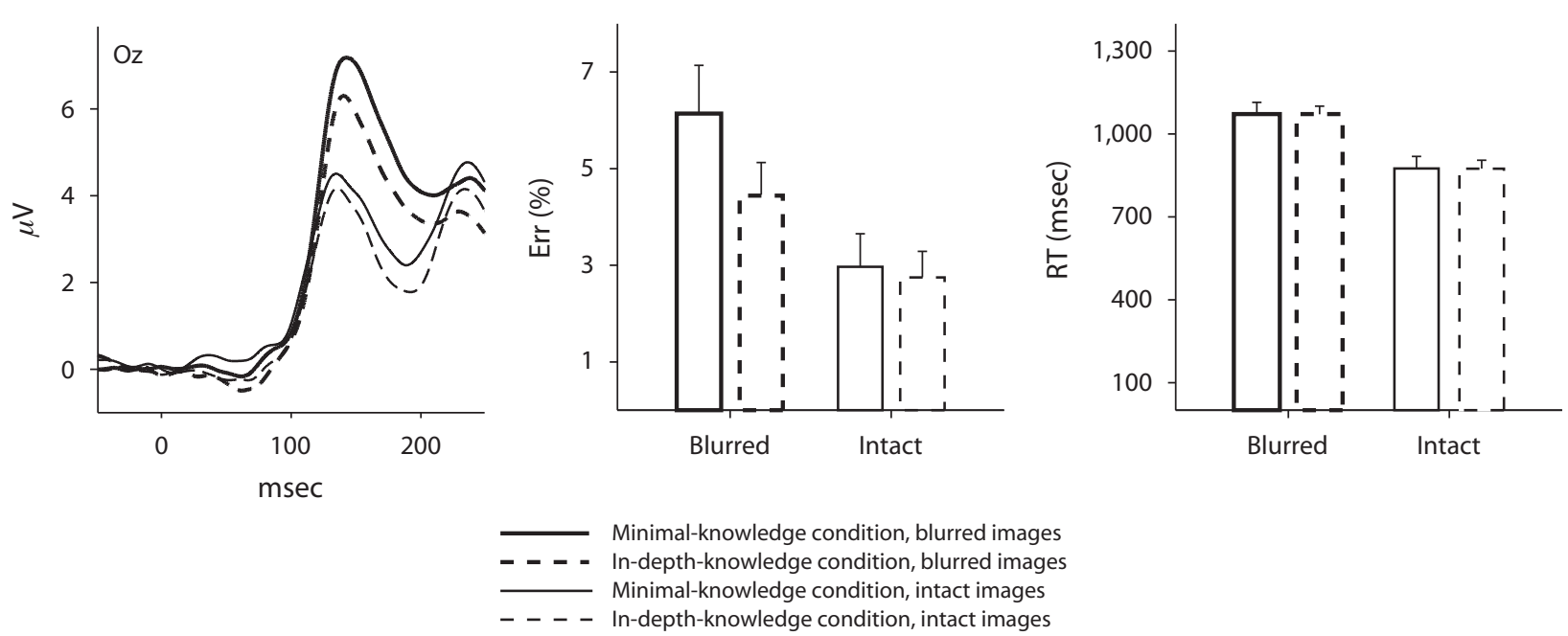

Figure 4. Effects of perceptual difficulty in Experiment 2 (blurred vs. intact images) and knowledge condition (in-depth vs. minimal knowledge). Event-related brain potentials (ERPs) are from an occipital electrode site (Oz, left panel). Effects on mean error rates (excluding omissions) (middle panel), and on mean response times (RTs) (right panel). Error bars depict standard errors of means.

the minimal and both the in-depth-knowledge condition $[F(55,2090)=8.84, p<.001]$, most pronounced at occipital electrodes $\mathrm{O} 1$ and $\mathrm{O} 2[F(1,38)=17.01, p<.001]$ (see Figure 3), and well-known objects $[F(55,2090)=8.0$, $p<.001 ; F(1,38)=6.3, p<.016$, at $\mathrm{O} 1$ and $\mathrm{O} 2]$. There was also a marginally significant difference between the in-depth-knowledge condition and well-known objects $[F(55,2090)=2.27, p=.046 ; F(1,38)=8.2, p<.018$ at $\mathrm{O} 1$ and $\mathrm{O} 2]$. Scalp distributions of knowledge effects (in-depth-knowledge minus minimal-knowledge condition) for the P1 and the N400 time segments did not differ $(F<0.5)$. Differences between groups in the N170 (see Figure 3; bottom), potentially reflecting effects of perceptual exposure, did not reach significance.

Experiment 1 indicates that in-depth knowledge affects ERPs in the time range of the P100 and the N400. Therefore, it appears that early perception is indeed affected by semantic knowledge.

Next, we probed the mechanisms underlying in-depthknowledge effects by manipulating a perceptual factor during testing.

\section{EXPERIMENT 2}

Object images were presented strongly low-pass filtered (blurred; see Figure 1) or in their original version. If in-depth knowledge influences visual perception, this effect should be enhanced when perceptual analysis is made more difficult by blurring. Therefore, we expected a stronger effect on P1 amplitude to blurred compared with intact images.

\section{Method}

Twenty new participants (15 women; mean age, 24.0 years; age range, 18-33) ran through the same procedure as did Group 2 of Experiment 1, except that during the test session all objects were first shown spatially low-pass filtered (Gaussian filter); thereafter images were presented again in intact versions.

\section{Results}

Figure 4 shows effects of knowledge and blurring on $\mathrm{P} 1$ amplitudes at an occipital electrode $(\mathrm{Oz})$ and on performance. An ANOVA with factors perceptual difficulty (blurred vs. intact images), task, and knowledge condition (in-depth vs. minimal knowledge) on peak amplitude of the $\mathrm{P} 1$ at $\mathrm{Oz}$ revealed main effects of perceptual difficulty $[F(1,19)=17.27, p<.001]$ and knowledge condition $[F(1,19)=17.09, p<.001]$, an interaction of task and perceptual difficulty $[F(2,38)=4.70, p<.01]$, and, most importantly, an interaction of perceptual difficulty and knowledge condition $[F(2,38)=9.44, p<.006]$. This interaction reflects stronger knowledge effects on P1 amplitude for blurred images.

In the N400 time window, an ANOVA yielded main effects of task $[F(110,2090)=31.57, p<.0001]$, perceptual difficulty $[F(55,1045)=2.99, p<.026]$, and knowledge condition $[F(55,1045)=8.14, p<.0001]$, and interactions of task and knowledge condition $[F(110,2090)=$ $7.74, p<.0001]$ and task and perceptual difficulty $[F(110,2090)=2.87, p<.031]$. In contrast to the $\mathrm{P} 1$ effects, however, there was no interaction of perceptual difficulty and knowledge condition $(F=0.5)$.

At variance with Experiment 1, an effect of knowledge on performance was found here. Relative to intact images, error rates (disregarding omissions) were increased in the blurred condition $[F(1,19)=20.81, p<.0001]$ and tended to be higher in the minimal- than in the in-depthknowledge condition $[F(1,19)=3.89, p=.063]$. There was no main effect of knowledge condition $(p=.18)$. Apart from main effects of blurring $[F(1,19)=243.3, p<$ $.001]$ and task $[F(2,19)=83.2, p<.001]$, there were no effects of minimal versus in-depth knowledge on RTs.

These results show that knowledge effects are perceptual effects. This holds even if blurring causes changes in energy levels or spatial frequency. In line with Bar et al. (2006), one might suggest that low spatial frequencies may 
be better tuned to activate early top-down modulations in vision. However, please note that we found no evidence of the frontal activation that Bar et al. assert.

\section{GENERAL DISCUSSION}

Exploring the effects of in-depth knowledge on object recognition, we obtained two main findings. A negative ERP deflection in the N400 time window gradually increased in amplitude from objects associated with minimal information, to those with newly acquired in-depth knowledge, to well-known objects. Because none of our tasks required the retrieval of in-depth knowledge, this effect reflects involuntary, automatic activation of facets of object meaning.

Furthermore, P1 amplitude was bigger for objects associated with minimal knowledge relative to both objects with in-depth knowledge and well-known objects. This suggests that stored in-depth knowledge shapes early stages of visual object perception, presumably by facilitating low-level visual analysis or reducing demands on detailed visual inspection, and that it does so in a direct and task-independent manner, even when this knowledge is irrelevant to the task at hand.

We propose two possible accounts for the present findings. First, high-level conceptual knowledge may exert a top-down influence on early perception, facilitating feature analysis by means of reentrant activation from higher level semantic to sensory cortical areas (e.g., Bar et al., 2006). In line with this assumption, recent evidence suggests that semantic information about objects is available very rapidly (Thorpe et al., 1996). Furthermore, although there were no behavioral knowledge effects in Experiment 1 , knowledge does seem to enhance correct object recognition when visual analysis is very demanding, as was the case in Experiment 2 with blurred images.

Second, the findings are also in line with suggestions that semantic knowledge is grounded in perception (Barsalou, 1999; Barsalou, Simmons, Barbey, \& Wilson, 2003; Goldberg, Perfetti, \& Schneider, 2006; Martin, 1998, 2007). Because in-depth knowledge was associated here with the meaning of visual object features, this knowledge might be stored within brain areas subserving visual analysis of these features, or may cause a restructuring of the neural networks in the perceptual system.

Alternatively, can the present results be accounted for by more global effects of selective attention? Because attention to the location or specific object features increases the P1 (e.g., Hillyard \& Anllo-Vento, 1998; Hopfinger, Luck, \& Hillyard, 2004), in-depth-knowledge effects might be due to differences in attention to specific features or whole objects. Since P1 amplitude was smaller in response to objects associated with in-depth knowledge, an attention-based account would have to postulate that knowledge decreases the need for allocating attention during perception.

This potential attention effect at testing cannot be due to learning-induced differences in visual experience on two grounds. First, objects had been familiarized before in-depth knowledge was provided; therefore, the initial learning conditions and familiarity levels were identical for all objects. Second, in Group 2 of Experiment 1, and in Experiment 2, object pictures had been absent while in-depth knowledge was acquired. This procedure precluded differences in visual inspection and attention during learning, leaving visual imagery during learning (a type of "priming") as the only possible difference between knowledge conditions. Because very similar P1 effects were obtained in the two groups, differential attentional influences of acquiring in-depth knowledge on perceptual experience are unlikely. Furthermore, during the test session of Experiment 1, objects associated with indepth knowledge were not processed any better or faster than were objects associated with minimal knowledge. This finding speaks against the idea that these objects are visually more familiar or learned with more focused attention.

The present results are therefore consistent with the assumption that knowledge shapes perception. As suggested above, this effect might be a consequence of top-down or, alternatively, perceptually grounded influences of semantic processes, possibly reducing the need to draw attention to perceptual analysis.

The suggested accounts are compatible with our observations concerning the underlying brain systems. First, knowledge-dependent modulations are closely linked to the visual processing modules active during the same time (P1 time window) and were influenced by a purely perceptual factor (blurring). Within the limits of spatial precision allowed by ERPs, free-fit dipole localization of the P1 and the knowledge effects yielded similar neuroanatomical loci (see Figure 5). Second, the brain systems related to the early effect appear to contribute substantially to the late effect in the N400 time window, yielding very similar scalp topographies (see Figure 2). Thus, in-depth knowledge influences visual systems early on, and continues to do so for several hundred milliseconds. Over this time period, semantic processing may become more fine grained, as suggested by the more graded knowledge effects in the later ERP timeframe.

Our findings extend those obtained with artificial objects and arbitrary semantic information to more heterogeneous common objects associated with coherent object-specific information. They also complement previous research on expertise in object recognition by elucidating the temporal dynamics of the effects of newly learned knowledge, independent of perceptual experience. We show that the nature of knowledge effects differs from the effects of perceptual expertise. Most strikingly, knowledge affects even earlier stages of visual analysis than perceptual expertise, as reflected in P1 modulations which precede perceptual expertise effects, most prominent in the N170 (e.g., Tanaka \& Curran, 2001), by about $40 \mathrm{msec}$.

In conclusion, the observed influence of in-depth knowledge on perception calls to mind a well-known quotation from the German poet and vision researcher Goethe (von Müller, 1982): “One only sees what one already knows 

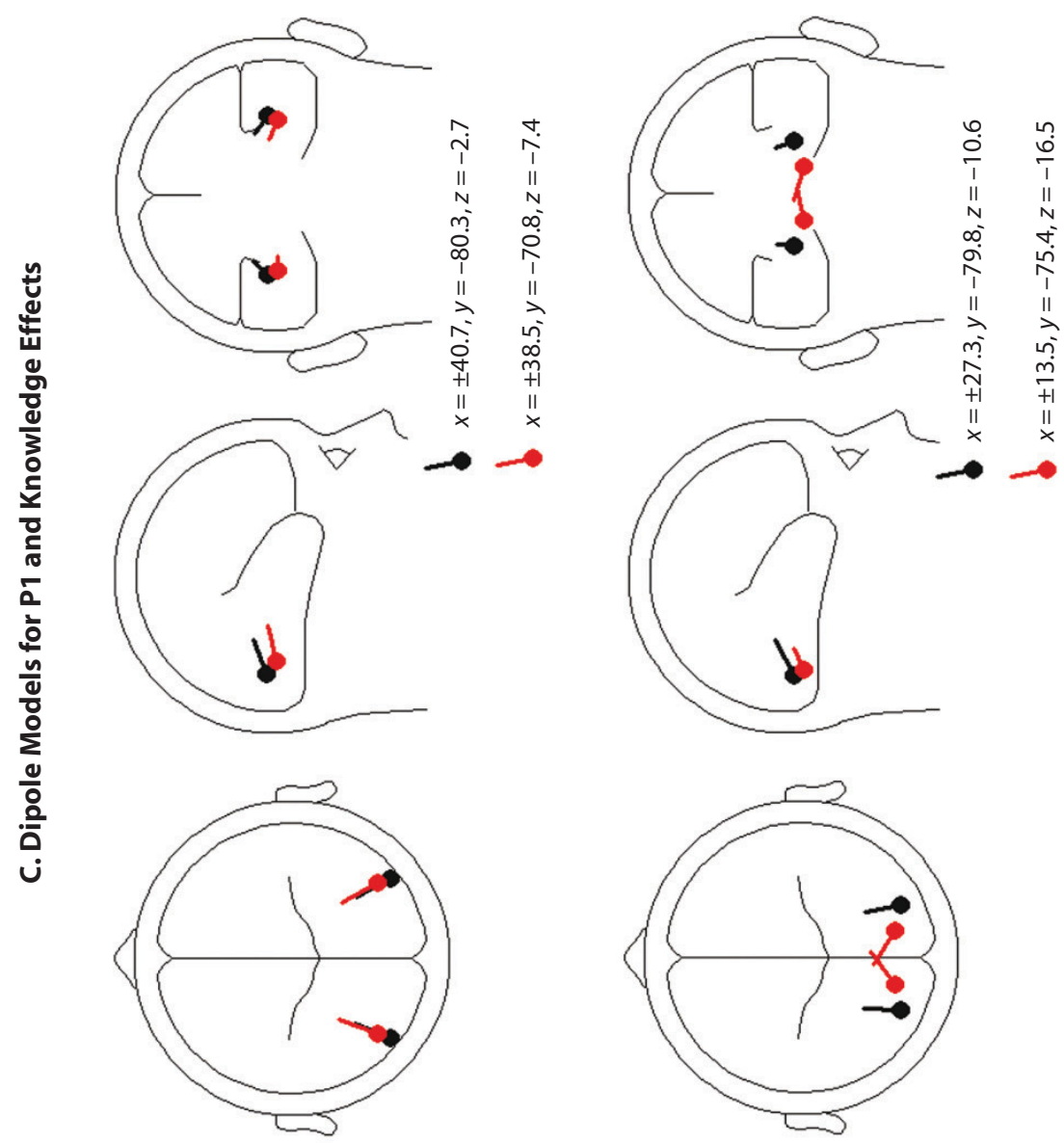

$\Xi \dot{0}$

施

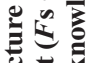

를 월

跣

造

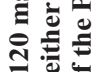

을

突

言苍

흘

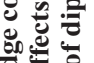

这

要

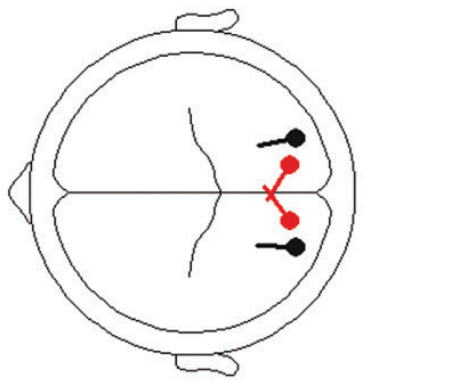

赵

要

西

웡

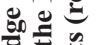

능

을

送

政

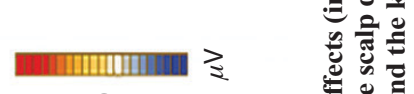
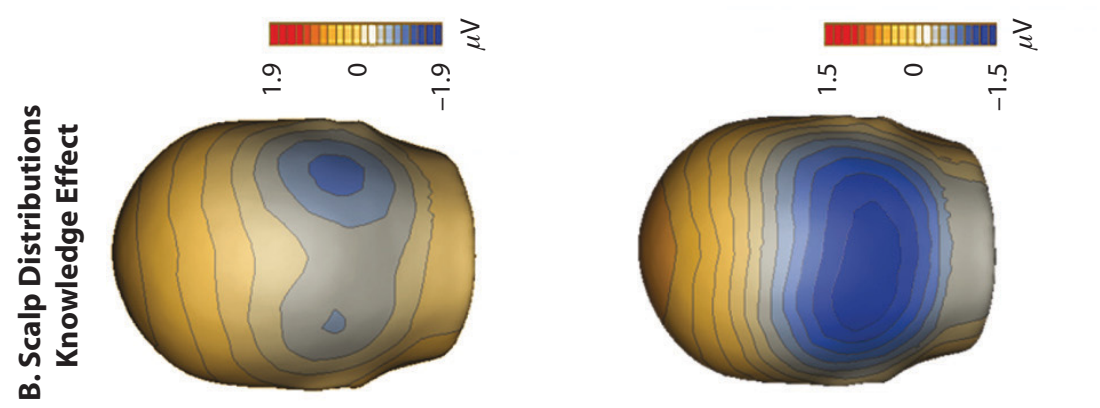

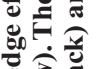

产

佂

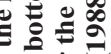

웅

政

跣

㯊

$\frac{10}{3} \div$
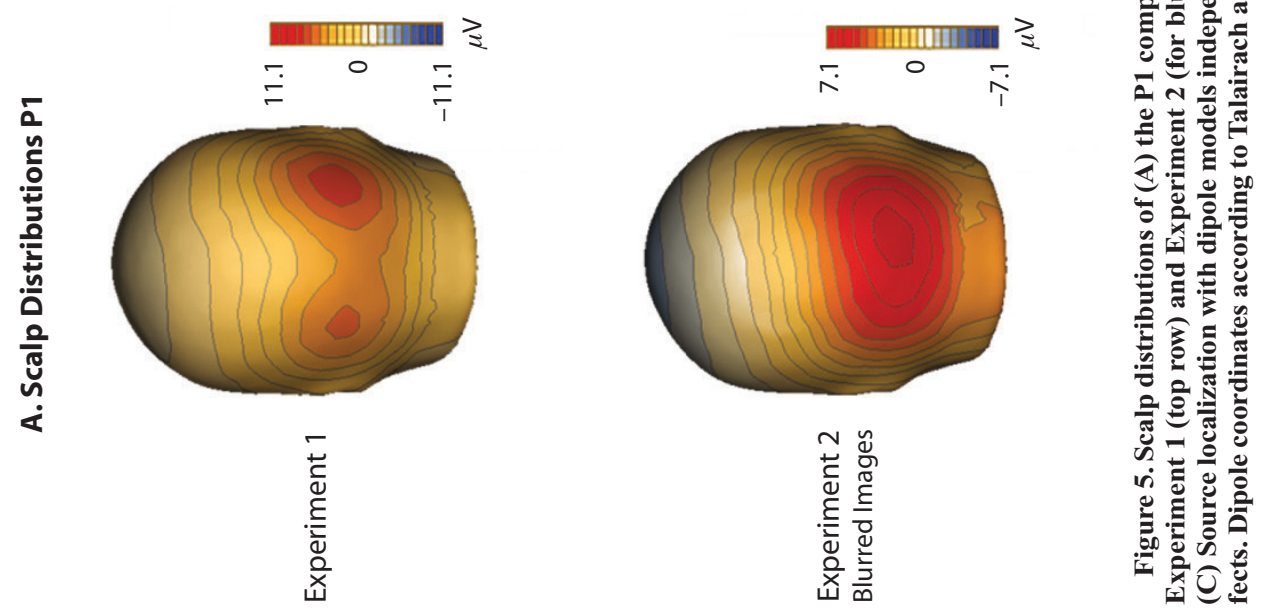
and understands. Often one will not discern aspects of objects encountered for many years until they become easily visible through maturing knowledge and education."

\section{AUTHOR NOTE}

This work was supported by German Research Foundation Grant AB277 to R.A.R. We thank G. Alpay, K. Hammer, J. Junker, R. Kniesche, T. Pinkpank, K. Unger, and D. Wisniewski for providing technical assistance or helping with data collection, O. Hohlfeld for finding the Goethe quotation, S. Neudecker from Zeit Wissen for making suggestions on how to find rare objects, and W. J. M. Levelt and A. Melinger for commenting on the manuscript. Correspondence concerning this article should be addressed to R. Abdel Rahman, Department of Psychology, Humboldt University, Rudower Chaussee 18, 10439 Berlin, Germany (e-mail: rasha.abdel.rahman@cms.hu-berlin.de).

\section{REFERENCES}

Bar, M., Kassam, K. S., Ghuman, A. S., Boshyan, J., Schmid, A. M., DALE, A. M., ET AL. (2006). Top-down facilitation of visual recognition. Proceedings of the National Academy of Sciences, 103, 449-454.

Barsalou, L. W. (1999). Perceptual symbol systems. Behavioral \& Brain Sciences, 22, 577-660.

Barsalou, L. W., Simmons, W. K., Barbey, A. K., \& Wilson, C. D (2003). Grounding conceptual knowledge in modality-specific systems. Trends in Cognitive Sciences, 7, 84-91.

BERG, P., \& Scherg, M. (1991). Dipole modelling of eye activity and its application to the removal of eye artefacts from the EEG and MEG Clinical Physiology \& Physiological Measurements, 12, 49-54.

Di Russo, F., Martínez, A., Sereno, M. I., Pitzalis, S., \& HillYARD, S. A. (2001). Cortical sources of the early components of the visual evoked potential. Human Brain Mapping, 15, 95-111.

Gauthier, I., James, T. W., Curby, K. M., \& Tarr, M. J. (2003). The influence of conceptual knowledge on visual discrimination. Cognitive Neuropsychology, 20, 507-523.

Gauthier, I., Skudlarski, P., Gore, J. C., \& Anderson, A. W. (2000) Expertise for cars and birds recruits brain areas involved in face recognition. Nature Neuroscience, 3, 191-197.

Gauthier, I., Tarr, M. J., Anderson, A. W., Skudlarski, P., \& Gore, J. C. (1999). Activation of the middle fusiform "face area" increases with expertise in recognizing novel objects. Nature Neuroscience, 2, 568-573.

Gauthier, I., Williams, P., TARr, M. J., \& TANaKa, J. W. (1998). Training "greeble" experts: A framework for studying expert object recognition processes. Vision Research, 38, 2401-2428.

Goldberg, R. F., Perfetti, C. A., \& Schneider, W. (2006). Perceptua knowledge retrieval activates sensory brain regions. Journal of Neuroscience, 26, 4917-4921

Grill-Spector, K., \& Kanwisher, N. (2005). Visual recognition: As soon as you know it is there, you know what it is. Psychological Science, 16, 152-160.

Hillyard, S. A., \& Anllo-Vento, L. (1998). Event-related brain potentials in the study of visual selective attention. Proceedings of the National Academy of Sciences, 95, 781-787.

Hopfinger, J. B., LuCK, S. J., \& Hillyard, S. A. (2004). Selective attention: Electrophysiological and neuromagnetic studies. In M. S. Gaz- zaniga (Ed.), The cognitive neurosciences (3rd ed., pp. 561-574). Cambridge, MA: MIT Press.

James, T. W., \& Gauthier, I. (2003). Auditory and action semantic features activate sensory-specific perceptual brain regions. Current Biology, 13, 1792-1796.

JAMES, T. W., \& GAUTHIER, I. (2004). Brain areas engaged during visual judgments by involuntary access to novel semantic information. $\mathrm{Vi}$ sion Research, 44, 429-439.

Kiefer, M., Sim, E.-J., Liebich, S., Hauk, O., \& TanaKa, J. (2007). Experience-dependent plasticity of conceptual representations in human sensory-motor areas. Journal of Cognitive Neuroscience, 19, 525-542.

Kutas, M., \& Hillyard, S. A. (1980). Reading senseless sentences: Brain potentials reflect semantic incongruity. Science, 207, 203-205.

Kutas, M., Van Petten, C. K., \& Kluender, R. (2006). Psycholinguistics electrified II: 1994-2005. In M. A. Gernsbacher \& M. Traxler (Eds.), Handbook of psycholinguistics (2nd ed., pp. 659-724). New York: Elsevier.

Lehmann, D., \& Skrandies, W. (1980). Reference-free identification of components of checkerboard-evoked multichannel potential fields. Electroencephalography \& Clinical Neurophysiology, 48, 609-621.

Martin, A. (1998). The organization of semantic knowledge and the origin of words in the brain. In N. Jablonski \& L. Aiello (Eds.), The origin and diversification of language (pp. 69-98). San Francisco: California Academy of Sciences.

Martin, A. (2007). The representation of object concepts in the brain. Annual Review of Psychology, 58, 25-45.

Meeren, H. K. M., van Heijnsbergen, C. C. R. J., \& de Gelder, B. (2005). Rapid perceptual integration of facial expression and emotional body language. Proceedings of the National Academy of Sciences, 102, 16518-16523.

Pexman, P. M., Hargreaves, I. S., Edwards, J. D., Henry, L. C., \& GoOdYEAR, B. G. (2007). The neural consequences of semantic richness: When more comes to mind, less activation is observed. Psychological Science, 18, 401-406.

TAlairach, J., \& Tournoux, P. (1988). Co-planar stereotaxic atlas of the human brain. New York: Thieme.

TANAKa, J. W., \& CurRan, T. A. (2001). A neural basis for expert object recognition. Psychological Science, 12, 43-47.

TanaKa, J. W., Curran, T. A., \& Sheinberg, D. L. (2005). The training and transfer of real-world perceptual expertise. Psychological Science, 16, 145-151.

Thorpe, S., Fize, D., \& Marlot, C. (1996). Speed of processing in the human visual system. Nature, 381, 520-522.

VON MÜLleR, F. T. A. H. (1982). Unterhaltungen mit Goethe [Conversations with Goethe]. Munich: Beck.

Weisberg, J., van Turennout, M., \& Martin, A. (2007). A neural system for learning about object function. Cerebral Cortex, 17, 513-521.

\section{NOTE}

1. Because average reference was used, only effects in interaction with electrode site are reported as "main effects" of the corresponding factors when all electrodes were included in the analysis.

(Manuscript received January 8, 2008; revision accepted for publication July 3, 2008.) 